Wright State University

CORE Scholar

2-1-1982

\title{
Magneto-Hall and Magnetoresistance Coefficients in Semiconductors with Mixed Conductivity
}

David C. Look

Wright State University - Main Campus, david.look@wright.edu

Follow this and additional works at: https://corescholar.libraries.wright.edu/physics

Part of the Physics Commons

\section{Repository Citation}

Look, D. C. (1982). Magneto-Hall and Magnetoresistance Coefficients in Semiconductors with Mixed Conductivity. Physical Review B, 25 (4), 2920-2922.

https://corescholar.libraries.wright.edu/physics/187

This Article is brought to you for free and open access by the Physics at CORE Scholar. It has been accepted for inclusion in Physics Faculty Publications by an authorized administrator of CORE Scholar. For more information, please contact library-corescholar@wright.edu. 


\title{
Magneto-Hall and magnetoresistance coefficients in semiconductors with mixed conductivity
}

\author{
D. C. Look
}

University Research Center, Wright State University, Dayton, Ohio 45435

(Received 1 September 1981; revised manuscript received 2 November 1981)

Magneto-Hall and magnetoresistance formulas, correct to order $B^{2}$, are derived for the case in which both single-carrier and mixed-carrier effects are important. Also, a new magneto-Hall coefficient is presented: $\beta=\left\langle\tau^{4}\right\rangle\langle\tau\rangle^{2} /\left\langle\tau^{2}\right\rangle^{3}-1$. Values of $\beta$ for various scattering mechanisms are calculated and compared with experiment.

The electron and hole currents in an isotropic semiconductor with spherical equal-energy surfaces can be written as ${ }^{1}$

$$
\begin{aligned}
& j_{n x}=\bar{\sigma}_{1 n} E_{x}-\bar{\sigma}_{2 n} E_{y}, \\
& j_{n y}=\bar{\sigma}_{2 n} E_{x}+\bar{\sigma}_{1 n} E_{y}, \\
& j_{p x}=\bar{\sigma}_{1 p} E_{x}+\bar{\sigma}_{2 p} E_{y}, \\
& j_{p y}=-\bar{\sigma}_{2 p} E_{x}+\bar{\sigma}_{1 p} E_{y},
\end{aligned}
$$

where the magnetic field $\vec{B}$ is in the $z$ direction and

$$
\begin{aligned}
& \bar{\sigma}_{1 n}=\frac{n e^{2}}{m_{n}^{*}}\left\langle\frac{\tau_{n}}{1+\omega_{c n}^{2} \tau_{n}^{2}}\right\rangle, \\
& \bar{\sigma}_{2 n}=\frac{n e^{2}}{m_{n}^{*}}\left\langle\frac{\omega_{c n} \tau_{n}^{2}}{1+\omega_{c n}^{2} \tau_{n}^{2}}\right\rangle .
\end{aligned}
$$

Similar equations for $\bar{\sigma}_{1 p}$ and $\bar{\sigma}_{2 p}$ follow by letting $n \rightarrow p$. Here $\omega_{c n}=e B / m_{n}^{*}$, all other symbols have their usual meanings, and the angular brackets denote averages over energy.

Consider first current in a single band, for example, the conduction band $(n>>p)$. Then, in accordance with the boundary condition $j_{n y}=0$, we get

$$
\sigma_{n} \equiv \frac{j_{n x}}{E_{x}}=\frac{\bar{\sigma}_{1 n}^{2}+\bar{\sigma}_{2 n}^{2}}{\bar{\sigma}_{1 n}}
$$

and

$$
R_{n} \equiv \frac{E_{y}}{j_{n x} B}=-\frac{1}{B} \frac{\bar{\sigma}_{2 n}}{\bar{\sigma}_{1 n}^{2}+\bar{\sigma}_{2 n}^{2}},
$$

where $R_{n}$ is the Hall coefficient. To order $\omega_{c n}^{2} \tau_{n}^{2}$ it can be shown ${ }^{1}$ that Eq. (3) yields

$$
\frac{\sigma_{n 0}-\sigma_{n}}{\sigma_{n 0}}=-\frac{\Delta \sigma_{n}}{\sigma_{n 0}} \simeq \frac{\Delta \rho_{n}}{\rho_{n 0}}=\xi_{n} R_{n 0}^{2} \sigma_{n 0}^{2} B^{2},
$$

where the subscript zero denotes measurement at zero magnetic field, $R_{n 0}=\left\langle\tau^{2}\right\rangle /\langle\tau\rangle^{2} n e$, and

$$
\xi_{n}=\frac{\left\langle\tau_{n}^{3}\right\rangle\left\langle\tau_{n}\right\rangle}{\left\langle\tau_{n}^{2}\right\rangle^{2}}-1
$$

Here

$$
\left\langle\tau_{n}^{q}\right\rangle=\frac{\int_{0}^{\infty} \tau_{n}^{q} \epsilon^{3 / 2} e^{-\epsilon / k T} d \epsilon}{\int_{0}^{\infty} \epsilon^{3 / 2} e^{-\epsilon / k T} d \epsilon}
$$

in the Boltzmann approximation.

Equation (5) is a well-known result. However, it has evidently also been commonly accepted that, to the same order of approximation $\left(\omega_{c n}^{2} \tau_{n}^{2}\right), R_{n}$ is independent of magnetic field. ${ }^{2}$ While this is true, strictly speaking, it is easily seen from Eq. (4) that a term of order $\omega_{c n}^{3} \tau_{n}^{3}$ (i.e., $\sim B^{3}$ ) in $\bar{\sigma}_{2 n} /\left(\bar{\sigma}_{1 n}^{2}\right.$ $+\bar{\sigma}_{2 n}^{2}$ ) will fall to order $B^{2}$ in $R_{n}$ because of the $B$ term in the denominator of Eq. (4). Thus, if we insert Eqs. 2(a) and 2(b) into Eq. (4), and carry out the averages to order $\omega_{c n}^{3} \tau_{n}^{3}$, the result is

$$
\frac{R_{n 0}-R_{n}}{R_{n 0}}=\frac{-\Delta R_{n}}{R_{n 0}}=\left(\beta_{n}-2 \xi_{n}\right) R_{n 0}^{2} \sigma_{n 0}^{2} B^{2},
$$

where

$$
\beta_{n}=\frac{\left\langle\tau_{n}^{4}\right\rangle\left\langle\tau_{n}\right\rangle^{2}}{\left\langle\tau_{n}^{2}\right\rangle^{3}}-1
$$

Some typical values of $\xi_{n}, \beta_{n}$, and $r_{n} \equiv\left\langle\tau_{n}^{2}\right\rangle /$ $\left\langle\tau_{n}\right\rangle^{2}$ are shown in Table I. Here the usual 
TABLE I. Theoretical values of $r_{n}, \xi_{n}$, and $\beta_{n}$ for several scattering mechanisms $\left(\tau_{n}=a_{n} \epsilon^{-s}\right)$, and experimental values for an $\mathrm{O}$-doped GaAs crystal.

\begin{tabular}{lccccc}
\hline \hline Type of scattering & $s$ & $r_{n}$ & $\xi_{n}$ & $\beta_{n}$ & $\beta_{n}-2 \xi_{n}$ \\
\hline $\begin{array}{c}\text { Acoustic deformation } \\
\text { potential }\end{array}$ & $\frac{1}{2}$ & 1.18 & 0.273 & 1.546 & 1.00 \\
$\begin{array}{c}\text { Acoustic piezoelectric } \\
\text { potential }\end{array}$ & $-\frac{1}{2}$ & 1.10 & 0.0865 & 0.268 & 0.0950 \\
$\begin{array}{l}\text { Ionized impurity } \\
\text { Experimental (Fig. 1) }\end{array}$ & $-\frac{3}{2}$ & 1.93 & 0.577 & 2.52 & 1.37 \\
\hline \hline
\end{tabular}

power-law energy dependence for $\tau_{n}$ is assumed;

i.e., $\tau_{n}=a_{n} \epsilon^{-s}$.

We now examine the case for mixed conductivity. Here ${ }^{1}$

$$
\begin{aligned}
& j_{x}=j_{n x}+j_{p x}=\left(\bar{\sigma}_{1 n}+\bar{\sigma}_{1 p}\right) E_{x}-\left(\bar{\sigma}_{2 n}-\bar{\sigma}_{2 p}\right) E_{y}, \\
& j_{y}=j_{n y}+j_{p y}=\left(\bar{\sigma}_{2 n}-\bar{\sigma}_{2 p}\right) E_{x}+\left(\bar{\sigma}_{1 n}+\bar{\sigma}_{1 p}\right) E_{y} .
\end{aligned}
$$

Again by setting $j_{y}=0$ we get

$$
\sigma \equiv \frac{j_{x}}{E_{x}}=\frac{\left(\bar{\sigma}_{1 n}+\bar{\sigma}_{1 p}\right)^{2}+\left(\bar{\sigma}_{2 n}-\bar{\sigma}_{2 p}\right)^{2}}{\left(\bar{\sigma}_{1 n}+\bar{\sigma}_{1 p}\right)}
$$

and

$$
R \equiv \frac{E_{y}}{j_{x} B}=-\frac{1}{B} \frac{\bar{\sigma}_{2 n}-\bar{\sigma}_{2 p}}{\left(\bar{\sigma}_{1 n}+\bar{\sigma}_{1 p}\right)^{2}+\left(\bar{\sigma}_{2 n}-\bar{\sigma}_{2 p}\right)^{2}}
$$

For $\tau_{n}, \tau_{p}$ independent of energy, well-known equations can be derived ${ }^{2,3}$ which are valid for arbitrary field strength, as long as $k T>>h \omega_{c}$. When $\tau_{n}$ and $\tau_{p}$ are energy dependent, however, we must again restrict ourselves to solving Eqs. (11) and (12) only to order $\omega_{c}^{2} \tau^{2}$. The results are

$$
\begin{aligned}
-\frac{\Delta \sigma}{\sigma_{0}} \simeq \frac{\Delta \rho}{\rho_{0}} & =\left[\frac{\sigma_{n} \sigma_{p}\left(R_{n} \sigma_{n}-R_{p} \sigma_{p}\right)^{2}}{\left(\sigma_{n}+\sigma_{p}\right)^{2}}+\frac{\xi_{n} R_{n}^{2} \sigma_{n}^{3}+\xi_{p} R_{p}^{2} \sigma_{p}^{3}}{\left(\sigma_{n}+\sigma_{p}\right)}\right]_{0} B^{2} \\
& =\left[\frac{c(\alpha b+1)^{2}}{\alpha^{2} b(1+b c)^{2}}+\frac{\xi_{n} b c}{1+b c}+\frac{\xi_{p}}{\alpha^{2} b^{2}(1+b c)}\right]_{0} r_{n 0}^{2} \mu_{n}^{2} B^{2}, \\
-\frac{\Delta R}{R_{0}}= & {\left[\frac{\sigma_{n}^{2} \sigma_{p}^{2}\left(R_{n}+R_{p}\right)\left(R_{n} \sigma_{n}-R_{p} \sigma_{p}\right)^{2}}{\left(\sigma_{n}+\sigma_{p}\right)^{2}\left(R_{n} \sigma_{n}^{2}+R_{p} \sigma_{p}^{2}\right)}+\frac{\beta_{n} R_{n}^{3} \sigma_{n}^{4}+\beta_{p} R_{p}^{3} \sigma_{p}^{4}}{R_{n} \sigma_{n}^{2}+R_{p} \sigma_{p}^{2}}-\frac{\left(2 \xi_{n} R_{n}^{2} \sigma_{n}^{3}+2 \xi_{p} R_{p}^{2} \sigma_{p}^{3}\right)}{\sigma_{n}+\sigma_{p}}\right]_{0}^{2} } \\
= & {\left[\frac{c(1-\alpha c)(1+\alpha b)^{2}}{\alpha^{2}(1+b c)^{2}\left(\alpha b^{2} c-1\right)}+\frac{\beta_{n} \alpha b^{2} c}{\left(\alpha b^{2} c-1\right)}-\frac{\beta_{p}}{\alpha^{2} b^{2}\left(\alpha b^{2} c-1\right)}-\frac{2 \xi_{n} b c}{(1+b c)}-\frac{2 \xi_{p}}{\alpha^{2} b^{2}(1+b c)}\right]_{0} r_{n 0}^{2} \mu_{n}^{2} B^{2}, }
\end{aligned}
$$

where $c=n / p, b=\mu_{n} / \mu_{p}, \alpha=r_{n} / r_{p}, \sigma_{n}=e n \mu_{n}$, $\sigma_{p}=e p \mu_{p}, R_{n}=-r_{n} / n e$, and $R_{p}=r_{p} / p e$. The subscript " 0 " on the large parentheses denotes that all quantities inside are to be evaluated at $B=0$. The first term in each of Eqs. (13) and (14) is clearly due to mixed-carrier effects alone, while the other terms involve single-carrier contributions.
Some data on a semi-insulating, O-doped GaAs crystal are presented in Fig. 1. For this sample $\rho_{0}=4.5 \times 10^{7} \Omega \mathrm{cm}, n_{H} \equiv 1 / R_{0} e=3.2 \times 10^{7} \mathrm{~cm}^{-3}$, and $R_{0} / \rho_{0}=4.3 \times 10^{3} \mathrm{~cm}^{2} / V$ sec. Two-carrier effects are not expected to be important since $b c$, $b^{2} c>>1$. Thus, Eqs. (5) and (8) should apply. Rather than plot $\Delta \rho / \rho_{0}$ and $-\Delta R / R_{0}$ vs $B^{2}$, we 


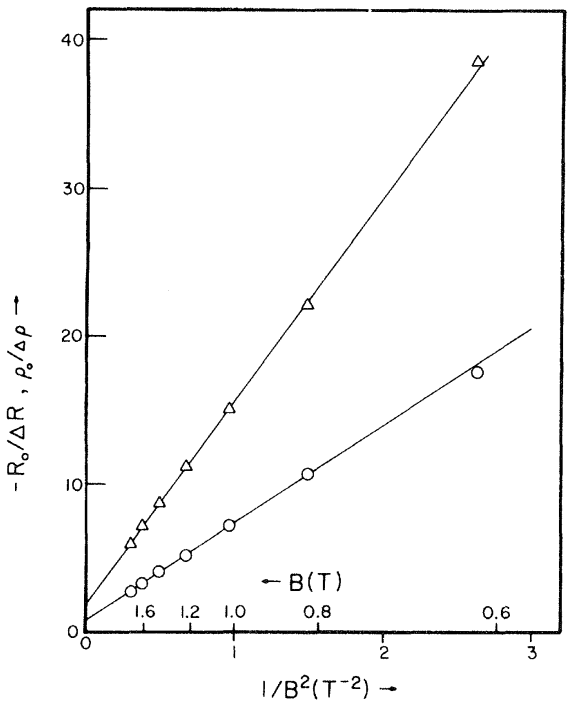

FIG. 1. Plots of $\rho_{0} / \Delta \rho$ (circles) and $-R_{0} / \Delta R$ (triangles) vs $1 / B^{2}$ for an O-doped, semi-insulating GaAs crystal at $296 \mathrm{~K}$.

have plotted $\rho_{0} / \Delta \rho$ and $-R_{0} / \Delta R$ vs $1 / B^{2}$ because the curves are then more linear. From such plots it is seen that the data seem to obey the relationships

$$
\begin{aligned}
& \frac{\rho_{0}}{\Delta \rho}=A_{\rho}+\frac{C_{\rho}}{B^{2}}, \\
& -\frac{R_{0}}{\Delta R}=A_{R}+\frac{C_{R}}{B^{2}},
\end{aligned}
$$

over the magnetic field range $6-18 \mathrm{kG}$. Here we would identify $C_{\rho}^{-1}=\xi_{n} R_{n 0}^{2} \sigma_{n 0}^{2}$ and $C_{R}^{-1}=\left(\beta_{n}\right.$ $\left.-2 \xi_{n}\right) R_{n 0}^{2} \sigma_{n 0}^{2}$. In fact, the slopes of the curves in Fig. 1 are the same as the initial slopes of $\Delta \rho / \rho_{0}$ and $-\Delta R / R_{0}$ vs $B^{2}$ plots, respectively, as they should be. Although it is not surprising that finite intercepts $\left(A_{\rho}\right.$ and $\left.A_{R}\right)$ exist at $1 / B^{2}=0$, the functional form represented by Eqs. (15) and (16) would not be expected to hold as this value were approached, i.e., as $B \rightarrow \infty$. That is, we cannot obviously identify $A_{\rho}$ and $A_{R}$ with the expected high-field values of $\rho_{0} / \Delta \rho$ and $-R_{0} / \Delta R$, respectively. Interestingly enough, the forms of Eqs. (15) and (16) hold exactly, for arbitrary $B$ (in the nonquantum limit, $k T>>\hbar \omega_{c}$ ), if single-carrier effects are totally absent. ${ }^{3}$ However, an analysis from this (mixed-carrier) point of view ${ }^{3}$ gives values of $\mu_{p}$ and $n_{i}$ (intrinsic concentration) which are much too high. In fact, GaAs samples with $\rho_{0} \leq 5 \times 10^{8}$ $\Omega \mathrm{cm}$ are not expected to have strong mixed-carrier effects. ${ }^{4}$ Thus, it appears that single-carrier (electron) effects dominate in this sample and that we can therefore obtain $\xi_{n}$ and $\beta_{n}-2 \xi_{n}$ from the slopes of the curves in Fig. 1.

The values obtained are listed in Table I. Although $\beta_{n}$ appears reasonable with respect to the calculated "pure-scattering" values, $\xi_{n}$ seems to be too high. It would be interesting to carry out a proper "mixed-scattering" energy averaging [Eq. (7)] of the various quantities in Eqs. (6) and (9). It would also be interesting to know how such factors as inhomogeneous compensation would affect the results.

For completeness, we also calculate the highfield $\left(k T>>\hbar \omega_{c}>>\hbar / \tau\right)$ limits of Eqs. (11) and (12), respectively:

$$
\begin{aligned}
& \sigma_{\infty}=\frac{e \mu_{n} \mu_{p}(n-p)^{2}}{n \mu_{p}\left\langle\tau_{n}\right\rangle\left\langle\tau_{n}^{-1}\right\rangle+p \mu_{n}\left\langle\tau_{p}\right\rangle\left\langle\tau_{p}^{-1}\right\rangle}, \\
& R=\frac{1}{e(p-n)} .
\end{aligned}
$$

The low-field limits, on the other hand, are

$$
\begin{aligned}
& \sigma_{0}=e\left(n \mu_{n}+p \mu_{p}\right), \\
& R_{0}=\frac{r_{p} p \mu_{p}^{2}-r_{n} n \mu_{n}^{2}}{e\left(n \mu_{n}+p \mu_{p}\right)^{2}},
\end{aligned}
$$

where $r_{n}$ and $r_{p}$ were defined previously. It is tempting to associate the intercept $A_{\rho}$ with $\sigma_{0} /\left(\sigma_{0}-\sigma_{\infty}\right)$, and $A_{R}$ with $R_{0} /\left(R_{0}-R \infty\right)$, but it must be remembered that Eqs. (15) and (16) do not hold theoretically over the entire range of $B$.

We would like to thank R.S. Allgaier for a helpful discussion.

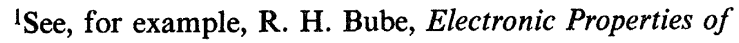
Crystalline Solids (Academic, New York, 1974), Chap. 10.

${ }^{2}$ See, for example, E. H. Putley, The Hall Effect and Semiconductor Physics (Dover, New York, 1968), p.
96.

${ }^{3}$ D. C. Look, J. Phys. Chem. Solids $\underline{36}, 1311$ (1975).

${ }^{4}$ D. C. Look, in Semi-Insulating III-V Materials, edited by G. J. Rees (Shiva, Orpington, 1980), p. 183. 